Topics

\title{
Long-Term Rearing of Medaka Aboard ISS to Clarify the Trans-Generation Effects in Vertebrates Induced by Cosmic Ray Irradiation
}

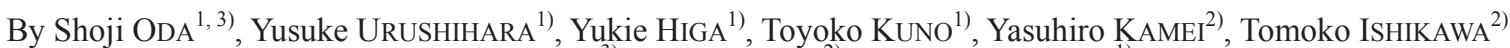 \\ Chiaki MUKAI ${ }^{3)}$, Takeshi TODO ${ }^{2)}$ and Hiroshi MITANI ${ }^{1)}$ \\ ${ }^{1)}$ Department of Integrated Biosciences, Graduate School of Frontier Sciences, The University of Tokyo, Kashiwa, Japan
${ }^{2)}$ Department of Radiation Biology and Medical Genetics, Graduate School of Medicine, Osaka University, Suita, Japan
${ }^{3)}$ Space Biomedical Research Office, Japan Aerospace Exploration Agency, Tsukuba, Japan
}

(Received August 18th, 2009)

\begin{abstract}
The effect of cosmic ray irradiation on astronaut health is a major problem to be addressed in the near future, however, it is not clear whether or not long-term irradiation of cosmic ray has trans-generation effects on astronauts. JAXA is developing an AQuatic Habitat system (AQH) that will enable us to keep more than 10 medaka adult fishes for three months on the ISS and we are going to investigate the effects of long-term cosmic ray irradiation on the genomes of vertebrate male germ cells using medaka. Transgenic (TG) medaka males that produce three-color fluorescent sperm are being produced. Cosmic-ray irradiation would induce deletion type DNA lesions and a sperm would lose fluorescence if the deletion occurs in the transgenes in the sperm genome. Because a male produces more than 100 million sperm, we can expect to investigate more than 300 million locus in germ cell genomes per one male fish and will be able to clarify whether long-term cosmic-ray irradiation aboard the ISS would induce serious effects on the vertebrate germ genomes or not and also whether micro gravity on the ISS would enhance mutation in vertebrate germ genomes. The first flight of the TG medaka fishes to the ISS will be in 2012 and will bring us the first data of the effects of long-term cosmic-ray irradiation on vertebrate germ genomes.
\end{abstract}

Key Words: Cosmic Ray, Transgeneration Effects, ISS, Medaka, Sperm

\section{Introduction}

1.1 Medaka as an experimental model animal for life science

Medaka (Oryzias latipes) is a small freshwater fish and native to Japan, South Korea, Taiwan and North-east of China. They are highly adaptive to a wide range of temperature, low $\mathrm{O}_{2}$ contents and can be reared with artificial foods in laboratories. These characters make medaka to be an excellent experimental animal for decades in physiology, developmental biology, genetics, cancer biology and radiation biology. In 2007, the draft genome sequence of medaka has been published ${ }^{1)}$ and opened to the public on web ${ }^{2)}$. The medaka genome project revealed that medaka and mammals share more than $70 \%$ of their genes, indicating that medaka and mammals share the basic body plans of the vertebrate. In addition, transgenesis (TG) in this species is easy, the protocol for in vitro fertilization (IVF) has been established and sperm of medaka can be cryo-preserved. Recently, targeted induced local lesions in genome (TILLING) is established and several gene knock-out medaka lines have been produced. Medaka is going to be a new model animal for functional genomics studies $^{3)}$.

1.2 A model animal is prerequisite for space medical science

Astronauts in the orbit are exposed to plenty of physiological stress. In the coming decade, more astronauts will "live" on the ISS for months or longer periods. Astronauts also will stay at the Moon base, and will go to the Mars via 3 years voyage in the deep space. In those situations, it is prerequisite to understand the mechanisms of the physiological stress on the astronauts in the space and to deal with it. Basic medical scientists will deal with these issues and they need some model animals in the orbit to investigate the physiological responses of astronauts in the space environment. Japan Aerospace Exploration Agency (JAXA) is developing the AQH system, which enable us to rear and keep small fishes like medaka on the ISS for up to 3 months.

\subsection{Biological effects of cosmic ray irradiation}

Measurements of radiation doses have shown that the dose of the cosmic ray irradiation on the ISS is more than 100 fold higher than that on the Ground and the astronauts are also exposed to high LET (Linear Energy Transfer) particles which would damage the cells inducing genomic instability in astronauts, although there is no reports on the serious affects of cosmic ray irradiation on the astronaut heath during/after a long stay on the ISS or the other space stations for months. These strongly suggest that human body is tolerant to the cosmic ray irradiation on the ISS even though the dose is higher than that on the Ground.

In 1997, Ikenaga et al. have reported that fruit flies Drosophila melanogaster loaded on a US space shuttle and irradiated with the cosmic ray for 8 days in the orbit showed the higher mutation frequency in the male reproductive cells and in contrast there was little difference in the mutation frequencies in the somatic cells between the flight flies and the ground control flies ${ }^{4}$. The dose of cosmic ray irradiation was very low to induce such a high frequency of mutations in the male reproductive cells and these results strongly suggest that the genetic effects of the cosmic ray irradiation on male 
reproductive cells could be greatly enhanced under the microgravity in the space. In their study, there was no difference in mutation frequencies of somatic cells between the flight flies and the control flies, suggesting that the cosmic ray irradiation has little effects on genomic stability in the somatic cells and this is consistent with a finding that the cosmic ray irradiation on astronauts has little effects on the health of the astronauts.

The study by Ikenaga et al. also arise a question whether or not the male reproductive cells of astronauts is also susceptible to the cosmic ray irradiation in the orbit. A vertebrate model animal which shares the molecular mechanisms of genetic stability in male reproductive cell system with human and can be used in the orbit is necessary to address this question. It is also unknown what kinds of physiological stresses will be given to astronauts and the model animal would be highly useful to investigate the physiological responses of vertebrates in the orbit.

In this project, we are going to establish medaka as an experimental model animal for basic medical researches on the ISS to investigate whether the cosmic ray irradiation affect on the genomic stability in the male reproductive cells in vertebrates or not.

\section{On-Going Projects}

\subsection{AQuatic Habitat (AQH): medaka keeping system on the ISS}

The AQH system will be equipped with 2 aquaria of about $700 \mathrm{ml}$ in volume and designed to keep 6 of adult fishes or 10 of fries of medaka in each aquaria. In the AQH, the fishes will be fed automatically with dried foods and kept for up to 3 months without maintenance by astronauts. Currently, fine tuning of the system is being processed to adapt a long period keeping of medaka on the Ground to launch the first medaka astronauts to the ISS in 2012.

The AQH is designed to rear and keep 12 to 20 medaka fishes on the ISS. Medaka can "live" in a small space; medaka can be kept in a small space for a long period and grown up to adults and spawn eggs in a small space. This is the most important feature of medaka as an orbital experimental animal because the space on the ISS is limited strictly.

\subsection{Detection of genomic mutation in transgenic medaka fishes with fluorescent sperm}

One male medaka provides more than 100 million sperm. Using the $\mathrm{AQH}$, we are going to investigate the effects of long-term cosmic ray irradiation on the genomes of vertebrate male reproductive cells. For this purpose, transgenic (TG) medaka fish lines that express fluorescent marker proteins in their sperm are being established. Vasa is well known to be expressed specifically in gonadal cells of Drosophila, fishes and mammals and the promoter of its medaka ortholog, olvas, can be used to label reproductive cells in vivo ${ }^{5)}$. We have found that sperm of the transgenic medaka which expresses green fluorescent protein (GFP) under the control by olvas promoter (olvas::GFP) are fluorescent. A new transgenic medaka line has been established on the base of T5 medaka strains which lacks 5 loci of body color determination genes $\left(b, g u, l f, w l, i^{b} ;^{6)}\right)$ and displays a "colorless" phenotype. T5 has been used for the specific locus assay to evaluate mutation frequency in male reproductive cells when irradiated with gamma-ray ${ }^{6}$. Males of this TG produce fluorescent sperm that express green fluorescent protein (GFP) in the cytosol. Males of another TG fish produce sperm that express yellow fluorescent protein (YFP) in their mitochondria, and males of the last $\mathrm{TG}$ fish will produce sperm that express red fluorescent protein (mCherry) in the sperm flagellum. A new TG medaka male that produces three-color fluorescent sperm will be produced by crossing the three TG fishes. Cosmic ray irradiation would induce deletion lesions of DNA, and a sperm would loss fluorescence if the deletion occurs in the transgenes in the sperm genome. Because a male produces more than 100 million sperm, we can expect to investigate more than 300 million locus in germ cell genomes per one male fish in this project and will be able to clarify whether long-term cosmic ray irradiation aboard the ISS would induce serious effects on the vertebrate germ genomes or not and also whether micro gravity on the ISS would enhance mutation in vertebrate germ genomes.

Medaka has been also used to investigate the effects of irradiation with gamma-ray ${ }^{7-9)}$ and high LET particle irradiation ${ }^{10)}$ on the genomic instability in male reproductive cells and a large amount of data have been accumulated. Gamma-ray irradiation also induced an obvious histological abnormalities in testis ${ }^{11,12)}$ and histological examination will be conducted to evaluate the effects of cosmic ray irradiation on male reproductive cell system in medaka. We have irradiated males of the TG, olvas::GFP, with the high LET carbon beam and found that the carbon beam would induce more severe histological abnormalities in the irradiated testis than gamma-ray irradiation of the identical dose.

2.3 Detection of genomic mutation in micro satellites by High-Resolution Melting analysis

High-Resolution Melting analysis is a new method for genotyping and is also going to be used to discover the mutations, detecting the difference between melting temperatures of homozygotic amplicons and heterozygotic amplicons ${ }^{13)}$. We are going to adapt the High-Resolution Melting analysis to detect mutations induced by gamma ray and high LET carbon beam irradiation in medaka male reproductive cells.

It is well known that the instability of microsatellites in genome is one of the main trans-generational effects of irradiation ${ }^{14)}$. We first screened the genomic database of an inbred medaka strain, Hd-rR, and found more than 10,000 microsatellites in medaka chromosome 9, which would be instable after gamma ray irradiation and selected 15 microsatellites for High-Resolution Melting analysis.

Males of an inbred medaka strain, Hd-rR, were irradiated and 1 month after the irradiation, each of them was mated with non-irradiated females of Hd-rR and fertilized eggs (more than 300 eggs per 1 male) were obtained. The embryos developed and 5 days after hatching, genomic DNA were extracted from the each fries. The High-Resolution Melting analysis on the 15 microsatellites revealed that the mutation frequency on these microsatellites induced 4 Gy gamma ray 
irradiation was about $1.5 \%$, while we did not found any mutations in non-irradiated control embryos.

\section{Perspective Remarks}

In the coming near future, human are going to stay in the ISS for months and, furthermore, human also have plans to stay on the Moon and to voyage to the Mars. In those days, astronauts would be exposed to the larger dose of cosmic ray irradiation and health care of those astronauts will be a major problem of space medicine, because the cosmic ray are different from any radiation rays used on the Ground experiments and we have no ways to evaluate their biological effects other than to expose the living organisms to the cosmic ray on the orbit.

To achieve the successful keeping of medaka on the ISS for 3 month, we have to understand what are the minimum essentials to keep medaka healthy in a small aquarium in the orbit. To address this question, we have to establish several technical protocols to keep medaka health in a small aquarium for months, in the next couple of year, and addressing to this question will also make medaka the first experimental model animal on the orbit for the space medical science.

\section{Concluding Remarks}

The first flight of the medaka to the ISS is currently planned to be in 2012 and will bring us the first data of the effects of long-term cosmic ray irradiation on vertebrate germ cell genomes. In this project, we are going to address the question whether the cosmic ray irradiation affect on the genomic stability in the male reproductive cells in vertebrates or not and the results of this project would be highly useful to evaluate the cosmic ray irradiation on male reproductive cells of astronauts.
K., Kato, T., Yamamoto, H. and Hara, R.:Mutations induced in Drosophila during space flight., Biol Sci Space, 11 (1997), pp.346-350.

5) Tanaka, M., Kinoshita, M., Kobayashi, D. and Nagahama, Y.:Establishment of medaka (Oryzias latipes) transgenic lines with the expression of green fluorescent protein fluorescence exclusively in germ cells: a useful model to monitor germ cells in a live vertebrate., Proc Natl Acad Sci U S A, 98 (2001), pp.2544-2549.

6) Shimada, A. and Shima, A.:High incidence of mosaic mutations induced by irradiating paternal germ cells of the medaka fish, Oryzias latipes., Mutat Res., 495 (2001), pp.33-42.

7) Shima, A., and Shimada, A.:The medaka as a model for studying germ-cell mutagenesis and genomic instability., Mar Biotechnol (NY), 3 (2001), pp.S162-167.

8) Shimada, A., Eguchi, H., Yoshinaga, S. and Shima, A.:Dose-rate effect on transgenerational mutation frequencies in spermatogonial stem cells of the medaka fish., Radiat Res, 163 (2005), pp.112.

9) Shimada, A. and Shima, A.:Transgenerational genomic instability as revealed by a somatic mutation assay using the medaka fish., Mutat Res, 552 (2004), pp.119-124.

10) Shimada, A., Shima, A., Nojima, K., Seino, Y. and Setlow, R. B.:Germ cell mutagenesis in medaka fish after exposures to high-energy cosmic ray nuclei: A human model., Proc Natl Acad Sci US A., 102 (2005), pp.6063-6067.

11) Kuwahara, Y., Shimada, A., Mitani, H. and Shima, A.:A critical stage in spermatogenesis for radiation-induced cell death in the medaka fish, Oryzias latipes., Radiat Res, 157 (2002), pp.386-392.

12) Kuwahara, Y., Shimada, A., Mitani, H. and Shima, A.:Gamma-ray exposure accelerates spermatogenesis of medaka fish, Oryzias latipes., Mol Reprod Dev, 65 (2003), pp.204-211.

13) Reed, G. H., Kent, J. O. and Wittwer, C. T.:High-resolution DNA melting analysis for simple and efficient molecular diagnostics., Pharmacogenomics, 8 (2007), pp.597-608.

14) Tsyusko, O., Yi, Y., Coughlin, D., Main, D., Podolsky, R., Hinton, T. G. and Glenn, T. C.:Radiation-induced untargeted germline mutations in Japanese medaka., Comp Biochem Physiol C Toxicol Pharmacol, 145 (2007), pp.103-110.

\section{References}

1) Kasahara, M., Naruse, K., Sasaki, S., Nakatani, Y., Qu, W., Ahsan, B., Yamada, T., Nagayasu, Y., Doi, K., Kasai, Y., Jindo, T., Kobayashi, D., Shimada, A., Toyoda, A., Kuroki, Y., Fujiyama, A., Sasaki, T., Shimizu, A., Asakawa, S., Shimizu, N., Hashimoto, S., Yang, J., Lee, Y., Matsushima, K., Sugano, S., Sakaizumi, M., Narita, T., Ohishi, K., Haga, S., Ohta, F., Nomoto, H., Nogata, K., Morishita, T., Endo, T., Shin-I, T., Takeda, H., Morishita, S. and Kohara, Y.: The medaka draft genome and insights into vertebrate genome evolution., Nature., 447 (2007), pp.714-719.

2) Ahsan, B., Kobayashi, D., Yamada, T., Kasahara, M., Sasaki, S., Saito, T., Nagayasu, Y., Doi K, Nakatani, Y., Qu, W., Jindo, T., Shimada, A., Naruse, K., Toyoda, A., Kuroki, Y., Fujiyama, A., Sasaki, T., Shimizu, A., Asakawa, S., Shimizu, N., Hashimoto, S., Yang, J., Lee, Y., Matsushima, K., Sugano, S., Sakaizumi, M., Narita, T., Ohishi, K., Haga, S., Ohta, F., Nomoto, H., Nogata, K., Morishita, T., Endo, T., Shin-I, T., Takeda, H., Kohara, Y. and Morishita, S.:UTGB/medaka: genomic resource database for medaka biology., Nucleic Acids Res, 36 (2007), pp.D747-D752.

3) Mitani, H., Kamei, Y., Fukamachi, S., Oda, S., Sasaki, T., Asakawa, S., Todo, T. and Shimizu, N.:The medaka genome: why we need multiple fish models in vertebrate functional genomics., Genome Dyn, 2 (2006), pp.165-182.

4) Ikenaga, M., Yoshikawa, I., Kojo, M., Ayaki, T., Ryo, H., Ishizaki, 